\title{
A comprehensive overview on genomically directed assembly of aromatic polyketides and macrolide lactones using fungal megasynthases
}

\begin{abstract}
Takayoshi Saruwatari ${ }^{1}$, Alex P Praseuth ${ }^{2}$, Michio Sato ${ }^{1}$, Kohei Torikai ${ }^{1}$, Hiroshi Noguchi ${ }^{1}$ and Kenji Watanabe ${ }^{1}$
Fungal polyketide synthases (PKSs) catalyze a carbon-carbon bond forming reaction in an iterative manner using a variety of acyl-CoA molecules as substrates when biosynthesizing complex polyketides. Although most members from this class of natural products exhibit notable biological activities, often they are naturally produced in trace levels or cultivation of the analyteproducing organism is less than feasible. Appropriately, to contend with the former challenge, one must identify any translational bottleneck and perform functional analysis of the associated enzymes. In recent years, many gene clusters purportedly responsible for biosynthesizing polyketides have been identified and cataloged from a variety of fungal genomes including genes coding for iterative PKSs, particulary bikaverin, zearalenone and hypothemycin biosynthetic enzymes. Mounting appreciation of these highly specific codons and their translational consequence will afford scientists the ability to anticipate the fungal metabolite by correlating an organism's genomic cluster to an appropriate biosynthetic system. It was observed in recent reports, the successful production of these recombinant enzymes using an Escherichia coli expression system which in turn conferred the anticipated metabolite in vitro. This review will focus on iterative PKSs responsible for biosynthesizing bikaverin, zearalenone and hypothemycin, and expand on befitting enzymatic reaction mechanisms and development of a highly versatile system that could potentially generate biologically active compounds.
\end{abstract}

The Journal of Antibiotics (2011) 64, 9-17; doi:10.1038/ja.2010.130; published online 24 November 2010

Keywords: biosynthesis; fungi; heterologous expression; iterative; PKS

\section{INTRODUCTION}

Polyketide synthase (PKS) is categorized according to the biosynthetic mechanisms they employ when assembling their secondary metabolites. Of the four categories, type I PKS can be described as a cluster of modular enzymes that catalyze a carbon-carbon bond forming reaction followed by a decarboxylative condensation to yield a polyketide backbone core structure. A variety of important natural products that exhibit antibiotic, immunosuppressive and antitumor activities have this type of core structure such as erythromycin, tacrolimus (FK506) and epothilone, respectively. Commonly, each type I PKS module contains a set of three essential subunits, ketosynthase, acyltransferase and acyl carrier protein, that work concertedly in catalyzing an alkyl chain extension reaction using only acyl-CoA as an extender unit (Figure 1). ${ }^{1-3}$

On the other hand, type II PKS is well known for producing aromatic metabolites. ${ }^{4}$ This class of PKS possesses a biosynthetic system that is analogous to the system described earlier with the exception of their ability to function as a standalone module and a relatively distinct catalytic mechanism from the modular PKS (Figure 2). Many biologically active natural products including tetracycline, tetracenomycin and daunorubicin synthesized by this family of enzymes were isolated from streptomycetes. The characteristic architecture of these compounds is comprised of acetyl- and malonyl-CoA. In addition, a ketosynthase/chain length factor has been reported to control the alkyl chain length that ultimately determines the number of aromatic rings in the final product.

In recent years, several genes coding for type III PKSs have been uncovered and successfully expressed in E. coli, particulary the chalcone synthase superfamily of enzymes (Figure 3). ${ }^{5,6}$ It was also observed and reported that these recombinant enzymes are capable of producing essential metabolites in vitro. Active sites of chalcone synthase, octaketide synthase and pentaketide chromone synthase have been studied extensively and rationally engineered for the purpose of biosynthesizing their analogs. As they are encoded in a single open reading frame and are post-translationally small in size, type III PKSs are ideal targets for protein engineering. The relative ease in which these genes are expressed makes molecular biological manipulation to obtain mutated enzymes more procurable and ameliorating the pursuit of understanding its biosynthetic pathway.

Moreover, biosynthetic gene clusters coding for iterative PKSs have been isolated and identified from fungi and has demonstrated the

\footnotetext{
${ }^{1}$ Department of Pharmaceutical Sciences, University of Shizuoka, Shizuoka, Japan and ${ }^{2}$ BioPharmaceuticals Formulation Development, Allergan, CA, USA Correspondence: Dr K Watanabe, School of Pharmaceutical Sciences, University of Shizuoka, Shizuoka 422-8526, Japan.

E-mail: kenji55@u-shizuoka-ken.ac.jp

Dedicated to the late Dr C Richard 'Dick' 'Hutch' Hutchinson for his exceptional contributions to natural product biosynthesis, engineering, and drug discovery.

Received 5 September 2010; accepted 13 October 2010; published online 24 November 2010
} 


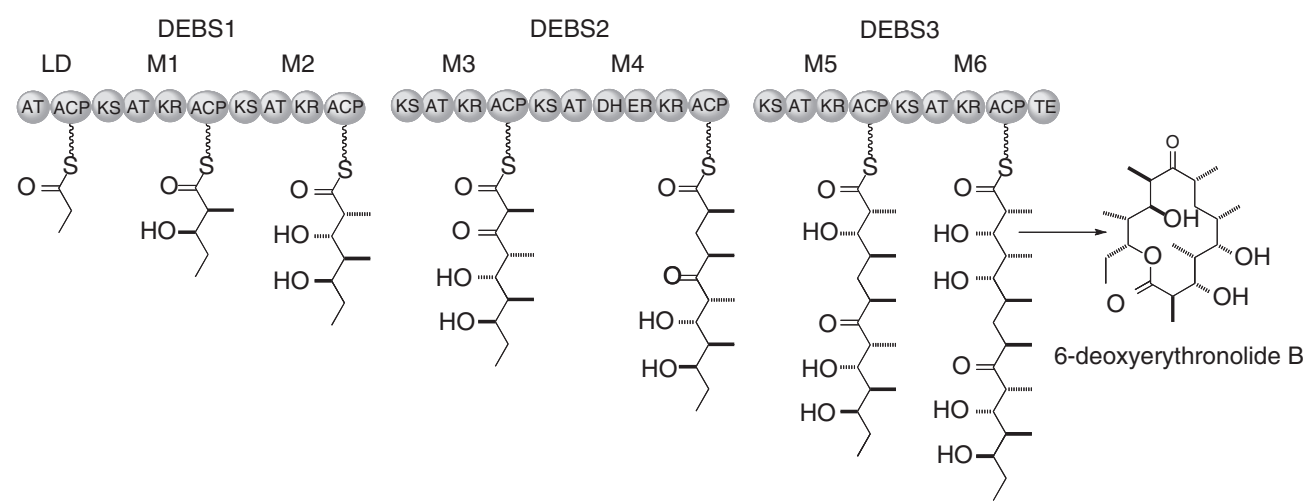

Figure 1 Organization of type I polyketide synthase. Biosynthesis of 6-deoxyerythronolide B, erythromycin aglycone. DEBS, 6-deoxyerythronolide B synthase; LD, loading domain; M, module; KS, ketosynthase; AT, acyltransferase; KR, ketoreductase; ACP, acyl carrier protein; DH, dehydratase; ER, enoylreductase; $\mathrm{TE}$, thioesterase.

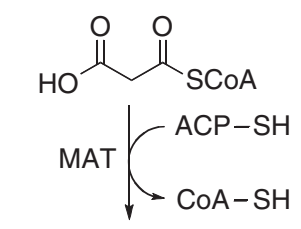

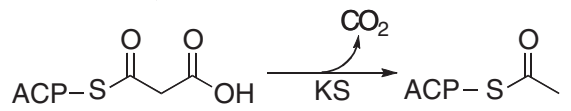

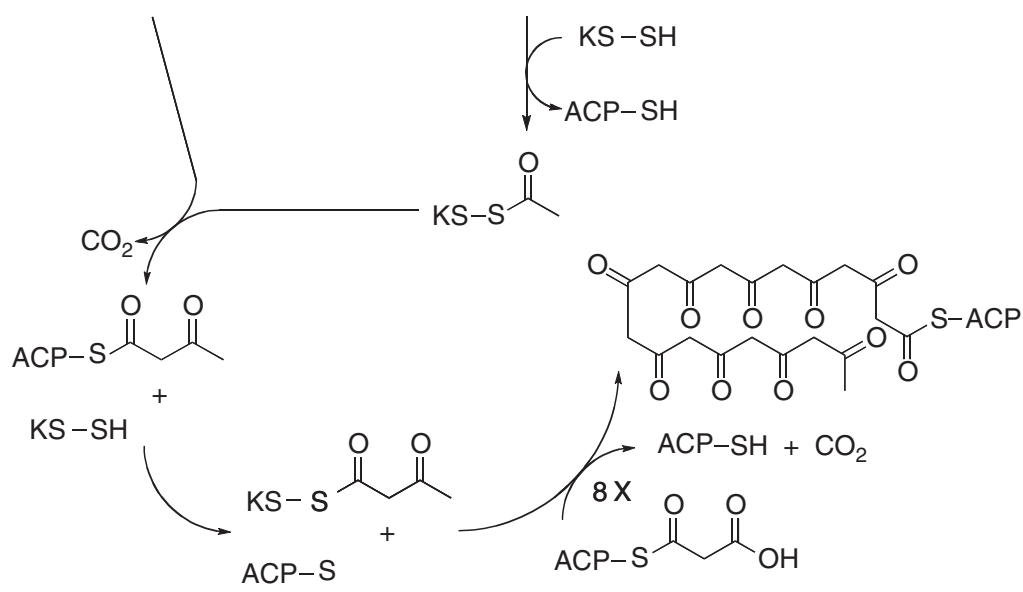
tetracenomycin F1

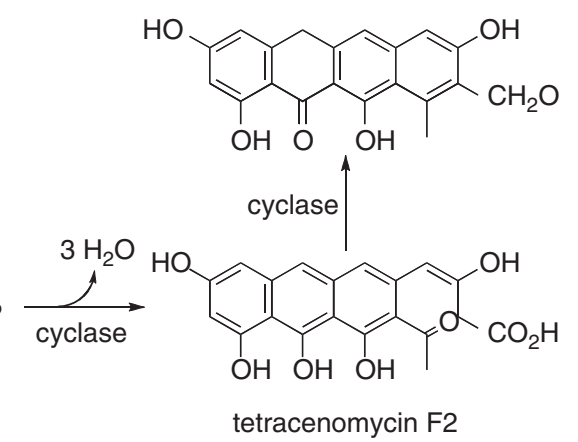

Figure 2 Organization of type II polyketide synthase. Biosynthesis of tetracenomycin F1. MAT, malonyl-CoA:ACP transacylase.

ability to produce a large number of bioactive molecules along with potential therapeutic agents as well as deadly mycotoxins such as aflatoxin. ${ }^{7,8}$ This class of enzymes uses an extender unit and assembly mechanism that is analogous to the characteristically modular type I PKS. Conversely, there are notable differences between the two systems, the iterative PKS is able to produce a straight chain polyketide that is later a substrate for a subsequent round of catalytic reaction using an additional acyl-CoA, typically malonyl-CoA, as a substrate by the single enzyme. According to its reduction domain activity, iterative PKS can be categorized into three subsections: a partially reducing, a non-reducing and a highly reducing PKS that biosynthesize 6-methylsalicylic acid, ${ }^{9}$ aflatoxin $\mathrm{B}_{1}{ }^{10}$ and lovastatin, ${ }^{11}$ respectively (Figure 4).

Aromatic polyketide 6-methylsalicylic acid is biosynthesized by ATX $^{12,13}$ encoding gene at $X$ that was discovered from Penicillium patulum. This gene was the first fungal gene to be expressed in streptomycetes ${ }^{14}$ and more recently, it was reported by Kealey and co-workers ${ }^{15}$ that at $X$ was successfully expressed in E. coli and Saccharomyces cerevisiae. While using S. cerevisiae as an expression host, it was observed that 6-methylsalicylic acid was produced at twice the level as that reported for the natural host. On the other hand, an E. coli expression system would offer additional advantages by facilitating genetic modification to the $a t X$ gene and strategic introduction of a chemical modification to the metabolite. Through detailed biochemical analysis, using recombinant enzyme expressed in E. coli, Fujii and co-workers demonstrated that 6-methylsalicylic acid was released by the dehydratase domain in ATX. ${ }^{9}$ These results underline the progress in fungal PKS research and utility of contemporary molecular biological tools by natural products scientists.

Cataloged as a non-reducing iterative PKS, PksA is responsible for synthesizing a potent hepatocarcinogen, aflatoxin. Townsend and co-workers were the first to successfully link the function of each PksA catalytic domain to the formation of the aromatic product 
a

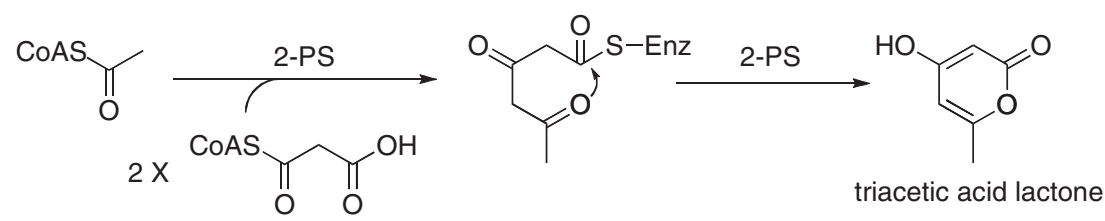

b<smiles>CC(C)S(=O)C(=O)CC(=O)O</smiles>

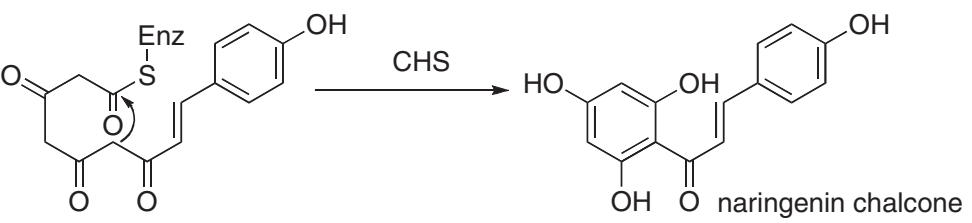

C<smiles>CC(C)[Si]S(=O)C(=O)CC(=O)O</smiles>

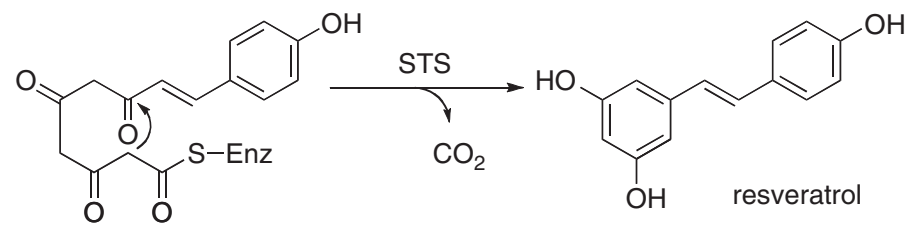

d

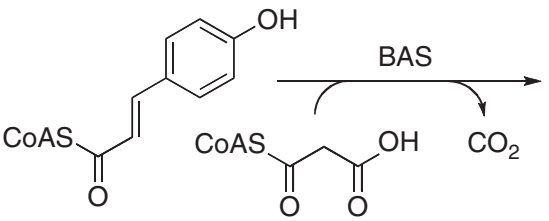<smiles>CC(=O)/C=C/c1ccc(O)cc1</smiles>

Figure 3 Chemical structures and biosynthetic schematics of type III polyketides, (a) triacetic acid lactone, (b) naringenin chalcone, (c) resveratrol and (d) benzalacetone. 2-PS, 2-pyrone synthase; CHS, chalcone synthase; STS, stilbene synthase; BAS, benzalacetone synthase.<smiles>Cc1cccc(O)c1C(=O)O</smiles>

6-methylsalicylic acid

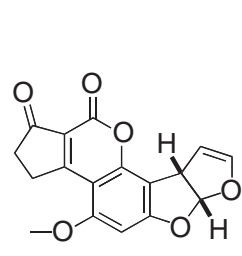

aflatoxin B1<smiles>CCC(C)C(=O)OC1CC(O)C=C2C=CC(C)C(CCC3CC(O)CC(=O)O3)C21</smiles>

lovastain
Figure 4 Chemical structures of fungal polyketides.

through domain dissection and reassembly. ${ }^{10,16}$ Moreover, the crystal structure of a product template (PT) domain commonly contained among this class of PKS was solved to $1.8 \AA$ resolution. ${ }^{17,18}$ According to these analyses, the enzyme is responsible for controlling explicit aldol cyclization and aromatization of incoming polyketide precursors.

Lovastatin is a pharmaceutically important compound because of its potent inhibitory activity toward 3-hydroxy-3-methylglutaryl-CoA reductase, the rate-limiting step of cholesterol biosynthesis. It is biosynthesized by the lovastatin nonaketide synthase accredited for producing lovastatin's decalin core structure via a Diels-Alder reaction originally reported by Hutchinson and Vederas and co-workers (Figure 5). ${ }^{11}$ Although lovastatin nonaketide synthase appears capable of biosynthesizing dihydromonacolin L via an endo Diels-Alder reaction, in vitro assays revealed such biosynthesis would require an additional enzyme, LovC. Therefore, no report has unambiguously demonstrated the ability for lovastatin nonaketide synthase to catalyze a Diels-Alder reaction as a single enzymatic reaction. Most recently, complete reconstitution of lovastatin nonaketide synthase's catalytic function has been reported in the presence and absence of cofactors (NADPH and SAM) and its partner enzyme, LovC. ${ }^{19,20}$

We focus here, on the iterative PKSs responsible for biosynthesizing bikaverin, ${ }^{21}$ zearalenone ${ }^{22,23}$ and hypothemycin ${ }^{24}$ and expand on befitting enzymatic reaction mechanisms and development of a highly versatile system that could potentially generate innovative biologically active compounds.

\section{AROMATIC POLYKETIDES BIOSYNTHESIZED USING PKS4 FROM GIBBERELLA FUJIKUROI}

To date, it has seemed difficult to accomplish a functional analysis of these iterative PKSs by way of in vitro assay because of the complication of heterologous expression of huge fungal gene coding for the PKS. Examples include pks4 gene, which was successfully expressed in E. coli through reconstitution of complementary DNA from PCR fragments using genomic DNA as a template. ${ }^{21}$ The translational product PKS4 is capable of producing aromatic polyketides using acylCoAs as substrates as reported by Tang et al. ${ }^{21}$ This PKS4 naturally found in G. fujikuroi is responsible for the biosynthesis of bikaverin (1, Figure 6), a fungal polyketide exhibiting anticancer activity. Mirroring the YWA1 biosynthetic pathway, ${ }^{25}$ a proposed mechanism for assembling the aromatic rings involves an aldol condensation reaction between $\mathrm{C} 2$ and $\mathrm{C} 7$ of the first ring followed by a Claisen condensation between $\mathrm{C} 10$ and $\mathrm{C} 1$ to form the pentaketide 1, 3, 6, 8-tetrahydroxynaphthalene ${ }^{26,27}$ scaffold by the thioesterase/Claisenlike cyclase (TE/CLC) domain in the enzyme which is finished off by a pyrone formation reaction. Subsequently, compound 2 is provided through a dehydration followed by cyclization between C12 and C17.

To demonstrate substrate tolerance and the ability to direct biosynthesis or induce metabolite divergence using the enzyme, this iterative PKS was incubated with malonyl-CoA as an extender unit 


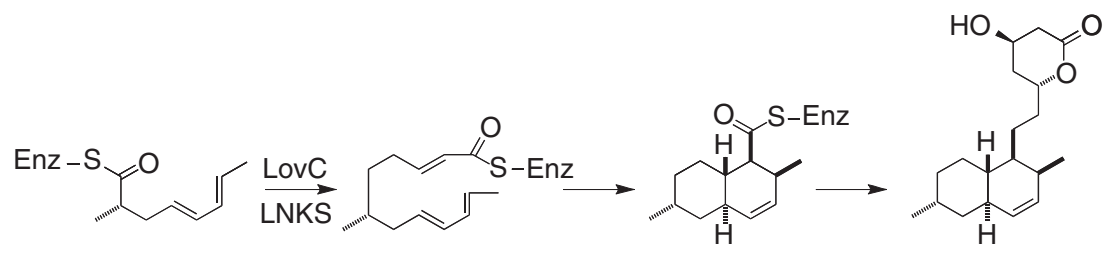

dihydromonacolin L

Figure 5 Reaction mechanisms of lovastatin nonaketide synthase (LNKS) and LovC.

\section{SAT KS MAT PT ACP TE/CLC}

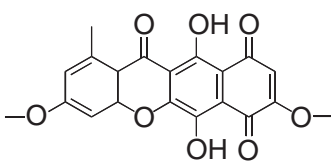

bikaverin1

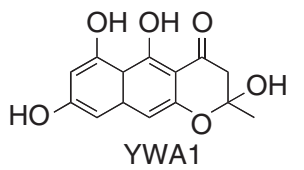

YWA1

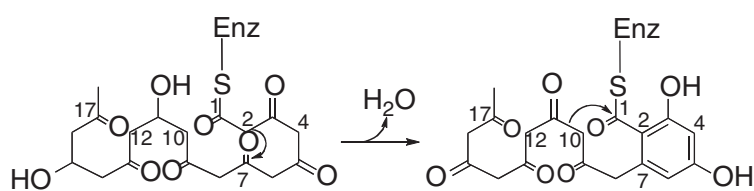<smiles></smiles>

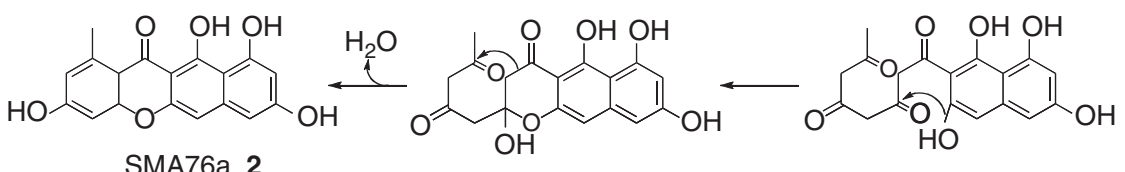

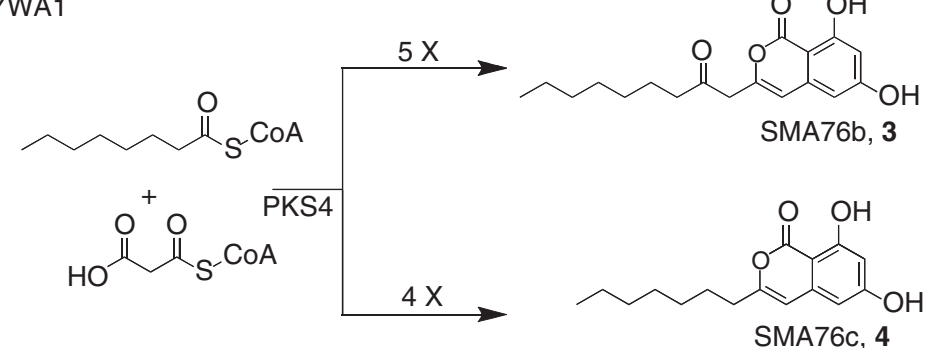

Figure 6 Biosynthesis of fungal polyketide by G. fujikuroi PKS4 and its catalytic domains.

and either its natural substrate malonyl-CoA or octanoyl-CoA as a starter unit. Surprisingly, the results of this experiment revealed the preference of PKS4 for octanoyl-CoA over malonyl-CoA as its starter unit, which is substantiated by the predominant level of $\mathbf{3}$ and $\mathbf{4}$ in vitro. A starter unit:acyl carrier protein transacylase (SAT) domain, commonly decides the priming unit during assembly of fungal polyketides. ${ }^{10}$ The SAT domain of PKS4 preferred malonyl-CoA, an unanticipated starter unit, opposed to its expected substrate, acylCoA. Containing a highly conserved cysteine residue in its active site, the amino acid sequence of the SAT domain is commonly described with a GXCXG motif. The consensus region in the active site can accept acyl-CoA as a starter unit, whereas GHSXG motif in the active site has an equivalent role for malonyl-CoA:acyl carrier protein transacylase (MAT). As the SAT domain of PKS4 does not contain the GXCXG motif, it has been suggested that an unknown mechanism for priming octanoyl-CoA could exist in the biosynthetic pathway.

Furthermore, mixing single-domain enzymes from PKS4 and type II PKS, revealed both distinct enzyme cross talk allowing biosynthesis of aromatic compounds along with regioselectively cyclization reaction. ${ }^{28}$ The results reported by Tang and co-workers showcased a $\beta$-ketoreductase (KR) domain of a bacterial PKS, actinorhodin biosynthetic enzyme, and its capacity to catalyze a reduction at C9 of a poly- $\beta$-keton core structure that is produced by a fungal PKS. Additionally, the stand-alone KR domain was able to produce $\mathbf{5}$ in

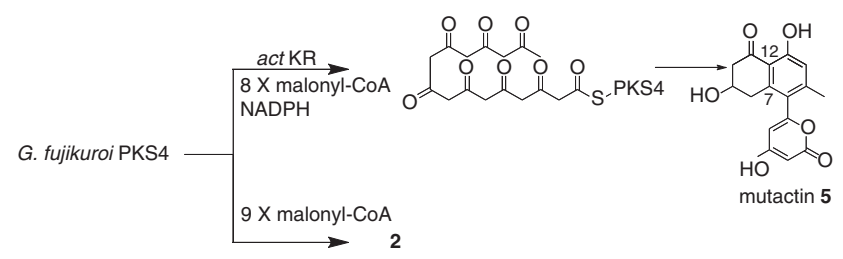

Figure 7 Biosynthesis of mutactin 5 by G. fujikuroi PKS4 and actinorhodin (act) KR type II PKS.

the absence of a starter unit that is pre-cyclized at the $\mathrm{C} 7-\mathrm{C} 12$ position (Figure 7). This corroborates the KR domain's ability to control the regioselective cyclization of C7-C12 with an aldol condensation during biosynthesis of 5 by PKS4 and actinorhodin KR as well as catalyzing the reduction. These remarkable results, exhibited by enzymes of this class, could provide an opportunity to vary the aromatic compounds of fungal polyketide scaffolds by means of blending both fungal and bacterial PKSs.

\section{RESORCYCLIC ACID LACTONES: ZEARALENONE AND HYPOTHEMYCIN}

In this section, we will continue to expand our review of fungal polyketides to include resorcylic acid lactones. At low concentrations, 
a<smiles>CC1CCCC(=O)CCC/C=C/c2cc(O)cc(O)c2C(=O)O1</smiles>

zearalenone<smiles></smiles>

hypothemycin<smiles>CO[C@@H](C)C[C@@H]1O[C@H]1/C=C\C=C/C(=O)Cc1c(Cl)c(O)cc(O)c1C(=O)O</smiles>

radicicol

b

G. zeae PKS4
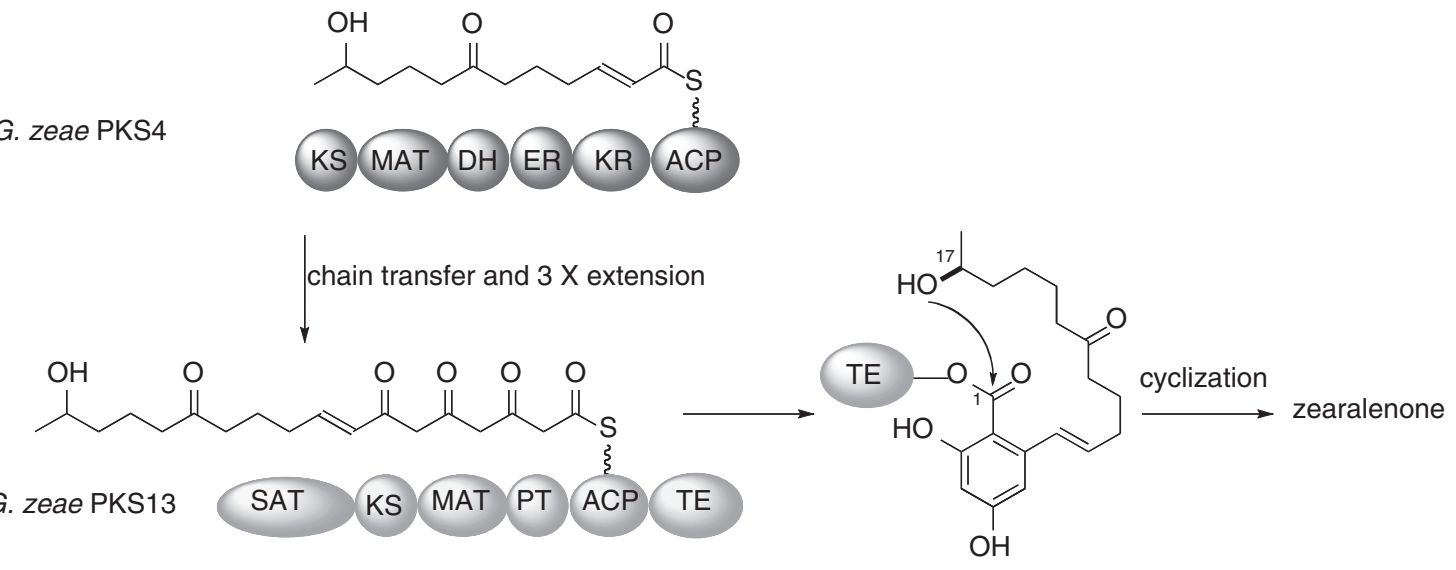

Figure 8 Representative resorcylic acid lactones (a) and a proposed biosynthetic mechanism of zearalenone (b).

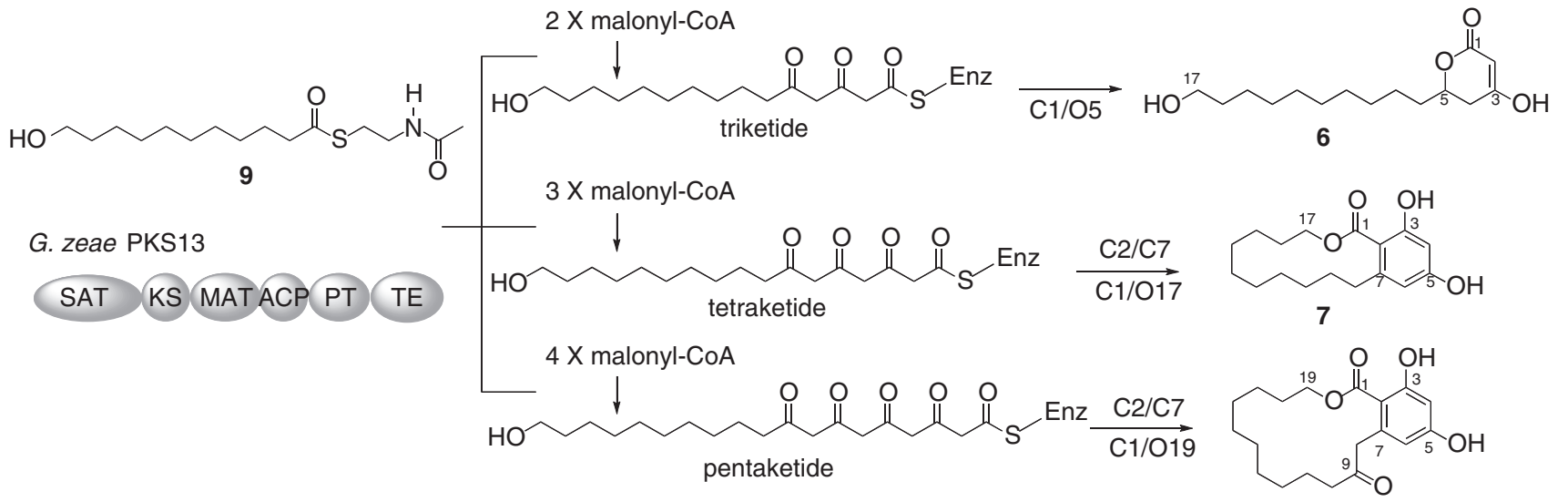

8

Figure 9 Proposed schemes of enzymatic synthesis of $\mathbf{6 , 7}$ and $\mathbf{8}$. Compound $\mathbf{6}$ is a triketide pyrone, compound $\mathbf{7}$ is a tetraketide resorcylic acid lactone and compound $\mathbf{8}$ is a pentaketide resorcylic acid lactone.

zearalenone, hypothemycin and radicicol have gained notoriety for exerting a variety of biological activity against mitogen-activated protein kinase (Figure 8). Initially isolated from Gibberella zeae, zearalenone's macrolide lactone structure assembly can be attributed to two iterative PKSs, suitably named PKS4 and PKS13 in the biosynthetic gene cluster. Analysis of amino acid sequence of both enzymes discerned PKS4 as a putative reducing PKS with its dehydratase, enoylreductase and KR domain, whereas PKS13 could potentially accept the ensuing straight-chain polyketide as a starter unit with its SAT domain. Understandably, PKS13 should be positioned in the rear of PKS4 and capable of catalyzing the necessary cyclization step when biosynthesizing the resorcylic acid lactone core. In studies using PKS13 that was heterologously expressed in E. coli, it was revealed that the enzyme's SAT domain was able to accept a broad range of substrates and was not partial to communicating with a variety of acyl carrier proteins. From the viewpoints presented, it is clear that the PKS biosynthetic machinery will consent to precursor-directed biosynthesis of metabolic analogs. 22

To reiterate the versatility of PKS13, three products, triketide pyrone 6, tetraketide resorcylic acid lactone 7 and pentaketide resorcylic acid lactone 8 (Figure 9), were successfully synthesized with malonyl-CoA as an extender unit and 11-hydroxyundecanyl- $N$-acetylcysteamine thioester (11-hydroxyundecanyl-SNAC, 9) as a starter unit which mimics a hexaketide primer naturally synthesized by PKS13. We can observe a gradual increase in the yield of $\mathbf{7}$ and $\mathbf{8}$ with a corresponding decrease in the yield of $\mathbf{6}$ upon increasing malonyl-CoA concentration. The results showed the ability of PKS13 to control the chain length by the abundance of the extender unit. Additionally, this enzyme showed 

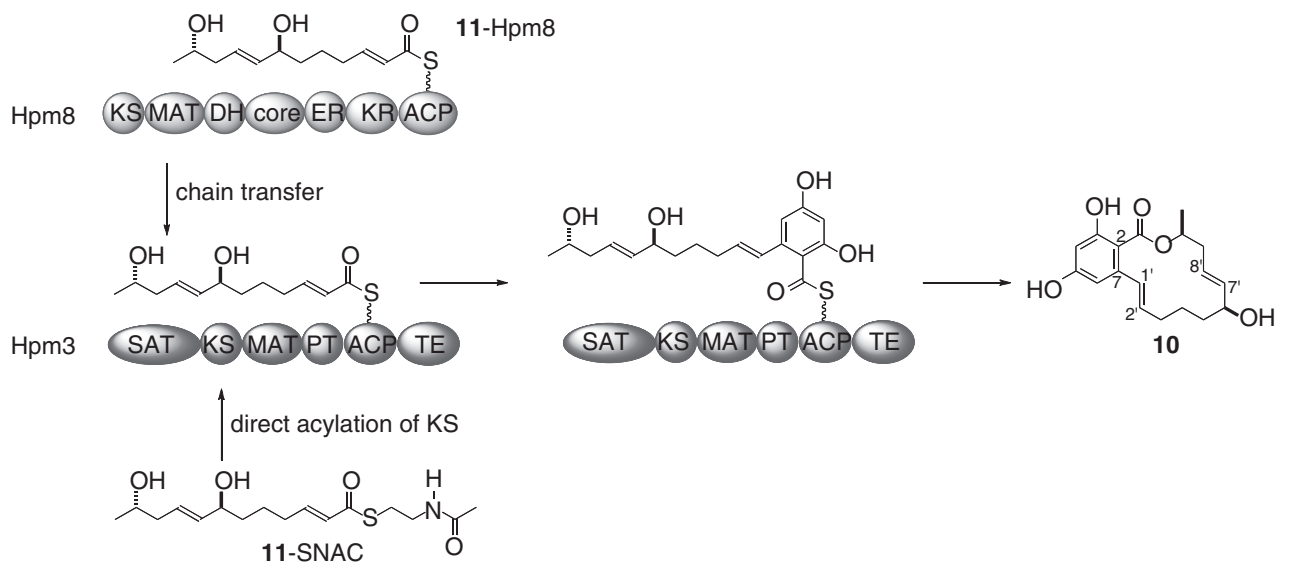

Figure 10 Biosynthesis of 10 by $\mathrm{Hpm} 8$ and $\mathrm{Hpm} 3$. Here, $\mathrm{Hpm} 3$ is shown accepting a hexaketide starter unit either from 11-Hpm8 via the SAT domain or from 11-SNAC directly.

broad substrate specificity toward CoA esters of C6 to C16 straightchain fatty acids, with decanoyl-CoA being the best substrate resulting in a higher abundance of $\alpha$-pyrones and resorcylic acids. Surprisingly, in vivo assays lacking exogenously supplied starter units resulted in productivity that was different from that observed in in vitro assays. The major products were synthesized with medium- and long-chain fatty acyl-CoAs as the starter units, which were present only in trace amounts in E. coli. It was postulated that these starter units were supplied to PKS13 with fatty acid synthase acyl carrier protein connecting with fatty acyl thioesters.

In this section, we will provide details on the reconstitution of two iterative PKSs, Hpm3 and 8, for in vitro biosynthesis of resorcylic acid lactone in hypothemycin biosynthesis (Figure 10). Two recombinant enzymes in combination (but neither enzyme alone) were reported to be responsible for biosynthesizing $\left(6^{\prime} S, 10^{\prime} S\right)$-trans- $7^{\prime}, 8^{\prime}$-dehydrozearalenol 10 in the $6^{\prime} R$ configuration. During the process, Hpm8 has been suggested in the biosynthesis to supposedly have a role of producing the starter unit, hexaketide ( $7 S, 11 S, 2 E, 8 E)$-7, 11-dihydroxydodeca-2, 8 -dienoate 11. The proposed function of Hpm3 is to accept 11 and extend to be a nonaketide, and then it catalyzes the regioselective cyclization and macrolactonization steps affording compound $10 .{ }^{24} \mathrm{To}$ understand the role of SAT domain in a biosynthesis of polyketide backbone structure, a hybrid PKS was constructed through the Hpm3 SAT domain replaced with the non-cognate PKS13 SAT domain. The hybrid iterative PKS was successfully expressed, and all the domains in the PKS exhibit activity by using 11-SNAC as a substrate. However, the $\mathrm{SAT}_{13}-\mathrm{Hpm} 3$ was not able to make a protein-protein interaction with $\mathrm{Hpm} 8$ during the production of 10. These results revealed that a cross talking between two iterative PKSs is controlled by a high specificity. As important findings, it may be possible to generate more diverse and novel compounds with an indispensable communication of an upstream iterative PKS and cognate SAT domain involved in a variety of downstream iterative PKSs accepting extender units.

\section{FUNCTIONAL ANALYSIS OF FUNGAL POLYKETIDE SYNTHASE PT DOMAINS}

The PT domain, called the PT domain for short, is generally contained in non-reducing iterative PKSs as a single domain. ${ }^{29}$ Functional analyses of the domain have indicated that the enzyme is able to control the regioselective cyclization of the polyketide backbone and govern the product's cyclic framework to acquire a C2-C7, C4-C9 or
C6-C11 configuration (Figure 11). A mechanistic study of PT domain from PksA has uncovered its involvement in tailoring the uncompleted polyketide backbone as reported by Townsend and co-workers. The domain can catalyze a regioselective cyclization of an octaketide via C4-C9 and C2-C11 aldol condensation to afford norsolorinic acid during biosynthesis of aflatoxin. Most recently, the PKS4 PT domain in the bikaverin PKS was evaluated to determine its role during the C2-C7 cyclization reaction when biosynthesizing a nonaketide SMA76 with the systematic removal of TE/CLC domain. The abridged enzyme, PKS4-99, confirmed the domain's function by producing a C2-C7 cyclized SMA93 12 and disentangling TE/CLC domain's role in the biosynthetic cascade as catalyzing a C1-C10 Claisen reaction. ${ }^{23,30}$ Furthermore, cleaving the PT domain from PKS4-99 provided atypical cyclic products $\mathbf{1 3}$ and $\mathbf{1 4}$ further confirming the domain's part in catalyzing the C2-C7 aldol condensation reaction. ${ }^{22}$ Cyclization of the C6-C11 position has also been reported and was found in the emodin biosynthetic pathway. A non-reducing iterative PKS, the atrochrysone carboxylic acid synthase is also able to synthesize a C6-C11 cyclized aromatic polyketide, atrochrysone. ${ }^{31}$ The biosynthetic enzymes catalyzing C6-C11 cyclization reaction to form these polyketides are normally the lack of a fused TE/CLC domain. The atrochrysone carboxylic acid synthase contains a $\beta$-lactamase-type TE domain instead of TE/CLC domain employed to release the product efficiently.

As we previously discussed, the PT domain could be a key player in constructing a fungal polyketide core structure. Therefore, fostering the predictability of a domain's route of cyclization by means of sequence homology will facilitate combinatorial biosynthesis and allow a breadth of novel molecules through a bioinformatics approach. Tang and co-workers analyzed 30 non-reducing iterative PKSs known to produce aromatic polyketides in attempts at establishing a relationship between a PT domain's protein sequence and its activities. Their findings ultimately categorized PT domains into five major groups (Figure 12).$^{30}$ Group I encompasses PKSs that biosynthesize single aromatic rings such as the ones for orsellinic acid and the aromatic portions of the resorcylic acid lactones. Depending on the primed starter unit, the PT domain is also capable of biosynthesizing a tetraketide backbone. Hence, these PT domains are able to regioselectively perform a C2-C7 cyclization reaction. Group II includes 1, 3, 6, 8-tetrahydroxynaphthalene synthases, a class of enzymes shown to catalyze the cyclization of a pentaketide backbone by way of a C2-C7 aldol condensation followed by a TE/CLC catalyzed 


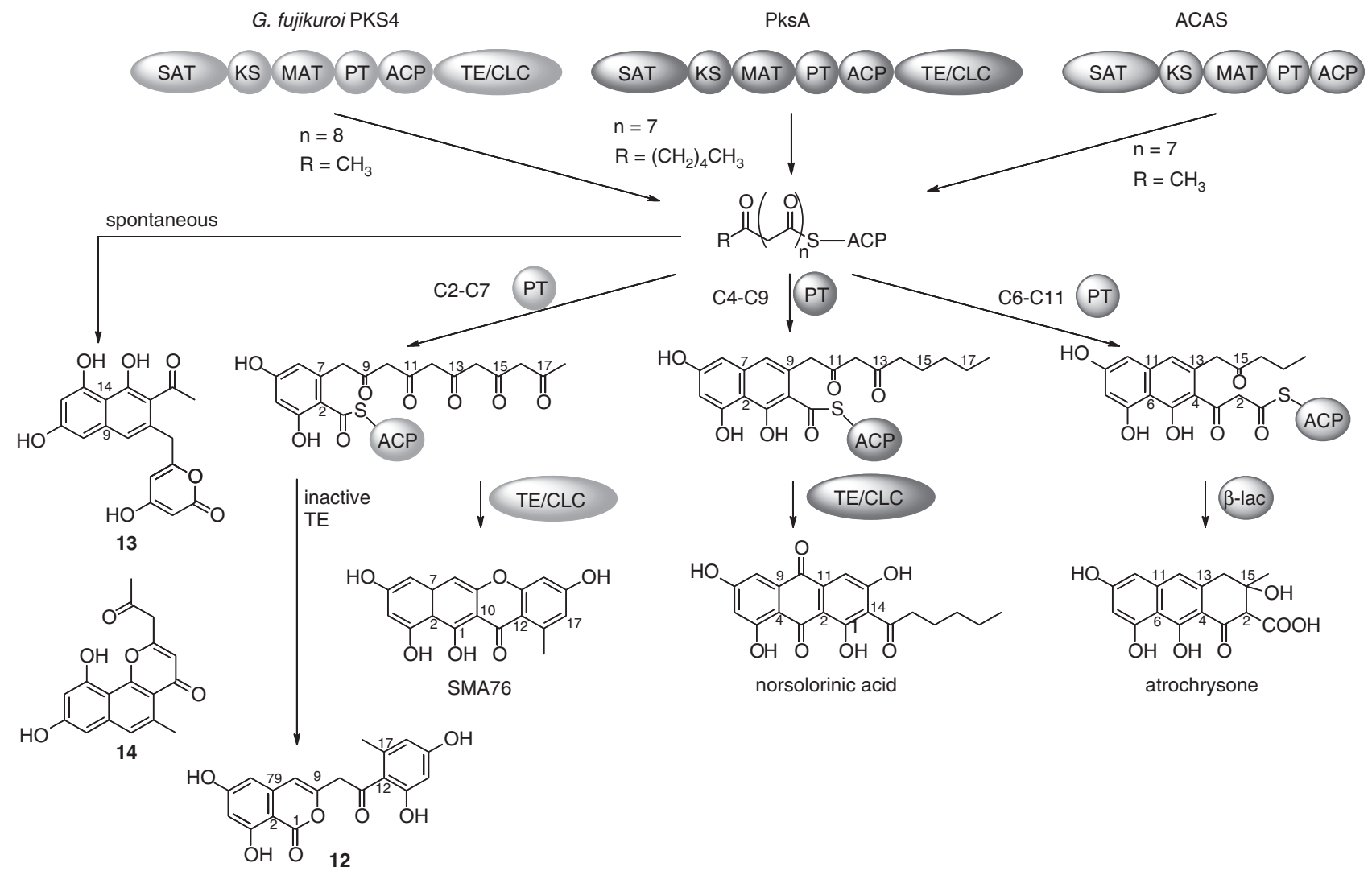

Figure 11 Biosynthesis of fungal aromatic polyketides. C2-C7, C4-C9/C2-C11 and C6-C11/C4-C13 are commonly observed to perform a cyclization pattern that is catalyzed by non-reducing iterative PKSs. Product template domains that mediate C2-C7 and C4-C9/C2-C11 folding modes have been characterized for PKS4 and PksA, respectively. TE/CLC domains catalyze the cyclization of the last ring and off-loading. In the absence of PT-mediated cyclization, the modest PKS4 spontaneously produces cyclized products 13 and 14.

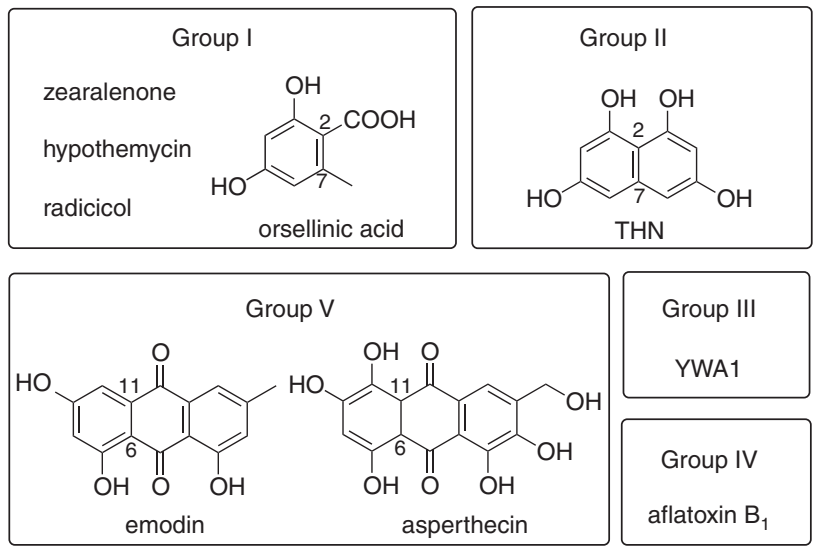

Figure 12 A major PKS group subdivided into five PT domains catalyzing the regioselective cyclization. The non-reducing iterative PKSs have been classified into five major groups: group I, C2-C7 monocyclic PKSs; group II, 1, 3, 6, 8-tetrahydroxynaphthalene (THN) synthases (C2-C7 bicyclic PKSs); group III, C2-C7 multicyclic PKSs; group IV, C4-C9 PKSs; and group V, C6-C11 PKSs.

cyclization of the second ring and concluding with release of the product. Group III, IV and V PKSs can accommodate a longer polyketide chain and structures containing multiple fused rings. PKSs in the third group regioselectively cyclizes its first ring via a C2-C7 cyclization mechanism identical to PKS4 and PKS12 in the zearalenone biosynthetic enzymes described in our previous sections. Much like PksA from the aflatoxin synthase biosynthetic cluster, group IV selectively aromatizes their product at the $\mathrm{C} 4-\mathrm{C} 9 / \mathrm{C} 2-\mathrm{C} 11$ position. Finally, Group V includes the asperthecin synthase and ASCS, which catalyzes the $\mathrm{C} 6-\mathrm{C} 11 / \mathrm{C} 4-\mathrm{C} 13$ regioselective cyclization reactions. As all members of this group lack C-terminal TE/CLC domains, all the enzyme-catalyzed cyclization reactions are managed by their PT domains. Interestingly, the enzymes discussed in this section could potentially provide a simple experimental platform to confirm the predicted regioselectivity of PT domains from non-reducing PKSs. The platform will also offer researchers a less cumbersome stage for rationally designing fungal polyketide analogs of varying chain length and ring assemblage by means of a simple mutation.

\section{CONCLUSION AND OUTLOOK}

In recent years, induced divergence of fungal metabolites by systematically swapping PT domains has been a topic of interest in the field of natural products research. By interchanging PKS4-99 and asperthecin synthase's implicit PT domain provided compound $\mathbf{1 5}$ (Figure 13) as its major product by incorporating a C6-C11 cyclization followed by a $\mathrm{C} 4-\mathrm{C} 13$ regioselective cyclization that is characteristic of PT domains from group V. Additionally, exchanging the PKS4-99 PT domain with an An03g05440 PT domain, a group III non-reducing iterative PKS encoded in Aspergillus nidulans genome, effectively produced an anticipated compound $\mathbf{1 2}$ as determined by the accessible domains and their respective catalytic mechanism (Figure 14). 

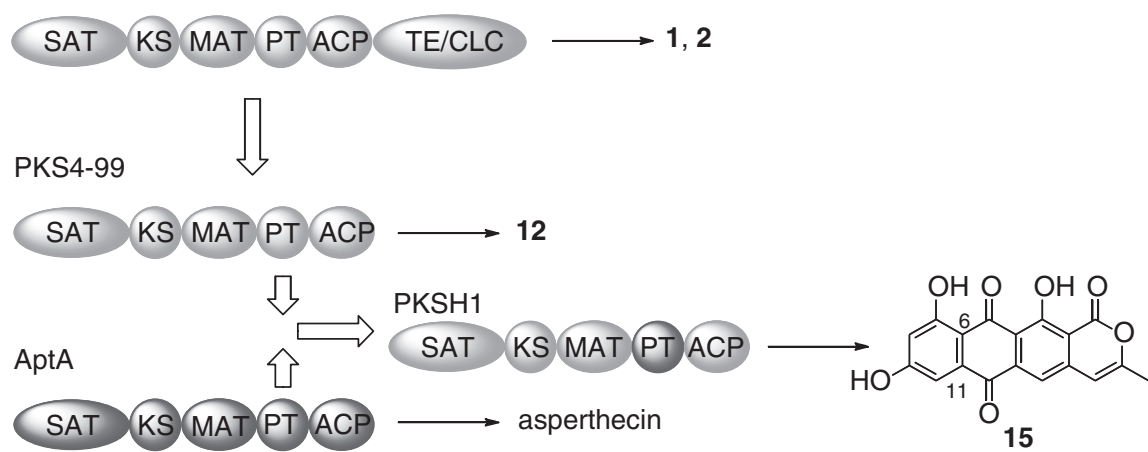

Figure 13 Construction of PKS4-99 and PKSH1 from two wild-type enzymes, PKS4 and AptA, and engineered biosynthesis of 12 and 15.

\section{PKS4-99}

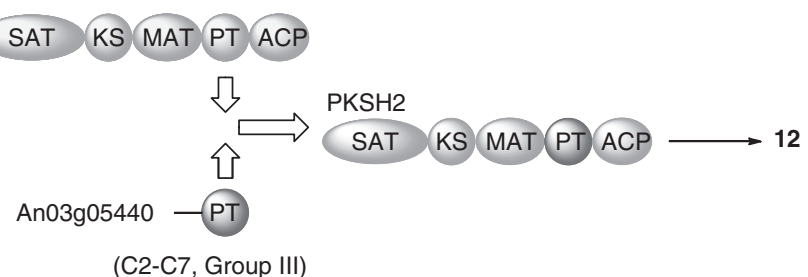

Figure 14 Construction of a composite enzyme, $\mathrm{PKSH} 2$, by interchanging PT domains of PKS4-99 and An03g05440, leading to the biosynthesis of 12.

Furthermore, recent elucidation of the crystal structure for PksA's PT domain has fostered increasing interest in this field of research by providing a more comprehensive representation of the enzyme. From the putative reaction mechanism and exploiting the straightforwardness of modifying a relatively short, single open reading frame coding for iterative PKSs, rationally engineering this family of protein has successfully provided a divergence of iterative polyketide scaffolds. Therefore, it is possible to generate more diverse and novel compounds with desirable pharmacological profiles through rational engineering of their biosynthetic machinery.

During the past decade, significant progress has been achieved by researchers to understand the fundamental aspects of secondary metabolite and how they are mechanistically produced by fungal polyketide. However, it still remains to simplify culturing conditions and isolation methodology of this class of natural products in the laboratory. In this regard, from the fungal genome project (http:// www.broadinstitute.org/), there could be a large number of biosynthetic gene clusters encoding putatively iterative PKSs in fungal genomic DNA that has yet to be functionally classified. Accordingly, future work in this field ought to concentrate on developing expression systems for heterologous production of complex polyketides. The splicing process is indispensable when generating complementary DNA and expressing fungal genomic DNA containing large genes at a range of $5-12 \mathrm{~kb}$ for heterologous production of secondary metabolites. To circumvent these obstacles, an expression system using yeast or fungus may prove advantageous. Advancement in genomic mining, ancillary to the approach described thus far will certainly provide prospects of isolating novel natural products by direct expression of fungal genomic DNA. These challenges, if accomplished, will arm us with innovative means of generating molecularly diverse fungal polyketides.

\section{ACKNOWLEDGEMENTS}

This paper is dedicated to the memory of Dr. Charles Richard Hutchinson for his pioneering studies in natural product biosynthesis, engineering and drug discovery. From the start of my vocation, he has lent his scientific expertise to mentor my research endeavors. With much appreciation, we would like to thank him for all his support. We would also like to express our appreciation to financial support from Japan Society for the Promotion of Science, Grant-inAid for Young Scientists (B) (No. 21780106) (KW) and by Industrial Technology Research Grant Program in 2009 (No. 09C46001a) from New Energy and Industrial Technology Development Organization (NEDO) of Japan (KW). These works were also supported in part by Kato Memorial Bioscience Foundation (KW), by Astellas Foundation for Research on Metabolic Disorders (KW) and by Hamamatsu Science and Technology Foundation (KW).

1 Cane, D. E., Walsh, C. T. \& Khosla, C. Harnessing the biosynthetic code: combinations, permutations, and mutations. Science 282, 63-68 (1998).

2 Shen, B. Polyketide biosynthesis beyond the type I, II and III polyketide synthase paradigms. Curr. Opin. Chem. Biol. 7, 285-295 (2003).

3 Khosla, C., Tang, Y., Chen, A. Y., Schnarr, N. A. \& Cane, D. E. Structure and mechanism of the 6-deoxyerythronolide B synthase. Annu. Rev. Biochem. 76, 195-221 (2007).

4 McDaniel, R., Welch, M. \& Hutchinson, C. R. Genetic approaches to polyketide antibiotics. 1. Chem. Rev. 105, 543-58 (2005).

5 Austin, M. B. \& Noel, J. P. The chalcone synthase superfamily of type III polyketide synthases. Nat. Prod. Rep. 20, 79-110 (2003).

6 Watanabe, K., Praseuth, A. P. \& Wang, C. C. A comprehensive and engaging overview of the type III family of polyketide synthases. Curr. Opin. Chem. Biol. 11, 279-286 (2007).

7 Watanabe, C. M., Wilson, D., Linz, J. E. \& Townsend, C. A. Demonstration of the catalytic roles and evidence for the physical association of type I fatty acid synthases and a polyketide synthase in the biosynthesis of aflatoxin B1. Chem. Biol. 3, 463-469 (1996).

8 Fujii, I. Functional analysis of fungal polyketide biosynthesis genes. J. Antibiot. 63, 207-218 (2010).

9 Moriguchi, T., Kezuka, Y., Nonaka, T., Ebizuka, Y. \& Fujii, I. Hidden function of catalytic domain in 6-methylsalicylic acid synthase for product release. J. Biol. Chem. 285, 15637-15643 (2010).

10 Crawford, J. M., Dancy, B. C. R., Hill, E. A., Udwary, D. W. \& Townsend, C. A. Identification of a starter unit acyl-carrier protein transacylase domain in an iterative type I polyketide synthase. Proc. Natl Acad. Sci. USA 103, 16728-16733 (2006).

11 Kennedy, J., Auclair, K., Kendrew, S. G., Park, C., Vederas, J. C. \& Hutchinson, C. R. Modulation of polyketide synthase activity by accessory proteins during lovastatin biosynthesis. Science 284, 1368-1372 (1999).

12 Moriguchi, T., Ebizuka, Y. \& Fujii, I. Analysis of subunit interactions in the iterative type I polyketide synthase ATX from Aspergillus terreus. Chembiochem 7, 1869-1874 (2006).

13 Moriguchi, T., Ebizuka, Y. \& Fujii, I. Domain-domain interactions in the iterative type I polyketide synthase ATX from Aspergillus terreus. Chembiochem 9, 1207-1212 (2008).

14 Bedford, D. J., Schweizer, E., Hopwood, D. A. \& Khosla, C. Expression of a functional fungal polyketide synthase in the bacterium Streptomyces coelicolor A3(2). J. Bacteriol. 177, 4544-4548 (1995) 
15 Kealey, J. T., Liu, L., Santi, D. V., Betlach, M. C. \& Barr, P. J. Production of a polyketide natural product in nonpolyketide-producing prokaryotic and eukaryotic hosts. Proc. Nat Acad. Sci. USA 95, 505-499 (1998).

16 Crawford, J. M., Thomas, P. M., Scheerer, J. R., Vagstad, A. L., Kelleher, N. L. \& Townsend, C. A. Deconstruction of iterative multidomain polyketide synthase function. Science 320, 243-246 (2008)

17 Crawford, J. M. et al. Structural basis for biosynthetic programming of fungal aromatic polyketide cyclization. Nature 461, 1139-1143 (2009).

$18 \mathrm{Paz}$ Kormana, T. et al. Structure and function of an iterative polyketide synthase thioesterase domain catalyzing Claisen cyclization in aflatoxin biosynthesis. Proc. Natl Acad. Sci. USA 107, 6246-6251 (2009).

$19 \mathrm{Ma}, \mathrm{S}$. M. et al. Complete reconstitution of a highly reducing iterative polyketide synthase. Science 326, 589-592 (2009).

20 Xie, X., Meehan, M. J., Xu, W., Dorrestein, P. C. \& Tang, Y. Acyltransferase mediated polyketide release from a fungal megasynthase. J. Am. Chem. Soc. 131, 8388-8389 (2009).

$21 \mathrm{Ma}, \mathrm{S} . \mathrm{M}$. et al. Enzymatic synthesis of aromatic polyketides using PKS4 from Gibberella fujikuroi. J. Am. Chem. Soc. 129, 10642-10643 (2007).

22 Zhou, H., Zhan, J., Watanabe, K., Xie, X. \& Tang, Y. A polyketide macrolactone synthase from the filamentous fungus Gibberella zeae. Proc. Natl Acad. Sci. USA 105, 6249-6254 (2008).

$23 \mathrm{Ma}$, S. M., Zhan, J., Xie, X., Watanabe, K., Tang, Y. \& Zhang, W. Redirecting the cyclization steps of fungal polyketide synthase. J. Am. Chem. Soc. 130, 38-39 (2008).
24 Zhou, H. et al. Enzymatic synthesis of resorcylic acid lactones by cooperation of fungal iterative polyketide synthases involved in hypothemycin biosynthesis. J. Am. Chem. Soc. 132, 4530-4531 (2010).

25 Fujii, I., Watanabe, A., Sankawa, U. \& Ebizuka, Y. Identification of Claisen cyclase domain in fungal polyketide synthase WA, a naphthopyrone synthase of Aspergillus nidulans. Chem. Biol. 8, 189-197 (2001).

26 Fujii, I., Mori, Y., Watanabe, A., Kubo, Y., Tsuji, G. \& Ebizuka, Y. Enzymatic synthesis of $1,3,6,8$-tetrahydroxynaphthalene solely from malonyl coenzyme $A$ by a fungal iterative type I polyketide synthase PKS1. Biochemistry 39, 8853-8858 (2000).

27 Fujii, I., Yasuoka, Y., Tsai, H. F., Chang, Y. C., Kwon-Chung, K. J. \& Ebizuka, Y. Hydrolytic polyketide shortening by ayg $1 \mathrm{p}$, a novel enzyme involved in fungal melanin biosynthesis. J. Biol. Chem. 279, 44613-44620 (2004).

28 Zhang, W., Li, Y. \& Tang, Y. Engineered biosynthesis of bacterial aromatic polyketides in Escherichia coli. Proc. Natl Acad. Sci. USA 105, 20683-20688 (2008).

29 Udwary, D. W., Merski, M. \& Townsend, C. A. A method for prediction of the locations of linker regions within large multifunctional proteins, and application to a type I polyketide synthase. J. Mol. Biol. 323, 585-98 (2002).

$30 \mathrm{Li}, \mathrm{Y} ., \mathrm{Xu}, \mathrm{W}$. \& Tang, Y. Classification, prediction, and verification of the regioselectivity of fungal polyketide synthase product template domains. J. Biol. Chem. 285, 2276422773 (2010).

31 Awakawa, T. et al. Physically discrete beta-lactamase-type thioesterase catalyzes product release in atrochrysone synthesis by iterative type I polyketide synthase. Chem. Biol. 16, 613-623 (2009). 